\title{
Addressing Cold Start Problem in Collaborative Filtering using Demographic Data with Entropy-based Methodology
}

\author{
*Victor T. Odumuyiwa and Olalekan P. Oloba \\ Department of Computer Sciences, University of Lagos, Lagos, Nigeria \\ vodumuyiwa@unilag.edu.ng I pet12003@yahoo.com \\ ORIGINAL RESEARCH ARTICLE \\ Received: 11-OCT-2021; Reviewed: 28-NOV-2021; Accepted: 22-DEC-2021 \\ http://dx.doi.org/10.46792/fuoyejet.v6i4.704
}

\begin{abstract}
Collaborative filtering-based recommender systems (RS) are faced with cold start problem. This problem arises when the RS does not have enough information or opinion about a person or about a product and therefore cannot make recommendation for such person. In this paper, the demographic data of the user such as age, gender, and occupation are utilized as additional sources together with existing users' rating to tackle the cold start problem by employing the entropy-based methodology to determine the degree of predictability. Experimental results on MovieLens dataset showed that the proposed method gives higher accuracy than other existing demographic-based methods.
\end{abstract}

Keywords- Cold Start, Collaborative Filtering, Demographic Approach, Entropy, Recommender Systems

\section{INTRODUCTION}

$\mathrm{T}$ The amount of information generated and available today is just overwhelmingly huge as compared to past years and this has given rise to a situation where it is becoming difficult to access relevant information from the severally available sources. The challenge therefore is how to quickly retrieve or access information that are relevant from this "information fog" which obviously comprises of both relevant and irrelevant details. It is illogical and if not impossible to go through all items to decide whether they are useful or not and thus finding relevant items has become more and more challenging. Recommender systems (RS) were conceived and created to solve this problem (Adomavicius \& Tuzhilin, 2005). Recommender systems are systems typically developed to assist people sift through available information in form of (and not limited to) books, movies, apps, videos, cars, articles, stories, webpages, friends, and jokes to quickly find the most interesting and relevant information and to filter out the less relevant or totally irrelevant ones.

In several sectors, such as the e-commerce, recommender systems have been used to model a customer's preferences and recommend things/items that they could like and find most useful to acquire. For example, Amazon.com is a well-known online store employs RS to recommend books to customers from the available books. Another popular e-commerce site is Moviefinder.com that uses RS to predict movies that a customer will like and recommends such to the customer. RS has also been applied to social coding platform to recommend developers for software development projects (Odumuyiwa \& Oyeyemi, 2019).

\section{*Corresponding Author}

Section B- ELECTRICAL/ COMPUTER ENGINEERING \& RELATED SCIENCES Can be cited as:

Odumuyiwa V.T. and Oloba O.P. (2021): Addressing Cold Start Problem in Collaborative Filtering using Demographic Data with Entropy-based Methodology, FUOYE Journal of Engineering and Technology (FUOYEJET), 6(4), 331-335. http://dx.doi.org/10.46792/fuoyejet.v6i4.704
Technically speaking, Recommender systems are systems that are used to generate meaningful recommendation of items or products that might be of interest to a user (Melville \& Sindhwani, 2017; Amatriain, Pujol \& Oliver, 2009) from a collection of items. The past two decades have witnessed an increase in researches dealing with automatic recommendation of items to people using different methods. These methods are generally classified into 3 broad categories (Adomavicius \& Tuzhilin, 2005) based on the approach they employ to perform recommendations: Collaborative filtering recommender systems (CFRS), Content based recommender (CBR) systems and Hybrid recommender systems.

In CFRS, the "wisdom of the crowd" is used to recommend items. Collaborative filtering (CF) typically associates a user to a cluster of similar users, and then proposes items liked by others in the cluster to the active user. CBR approaches, on the other hand, suggest items that a user has previously liked and/or items that are matched to the profile of a user. Hybrid systems methods combined both CF and CBR systems for recommendation.

CF methods can be broken down into two major categories: Neighbourhood-based and Model-based approaches (Bell, Koren \& Volinsky, 2007; Herlocker, Konstan \& Riedl, 2002). Neighbourhood-based method provides recommendation by building similarities between users (neighbours) using users' ratings. Recommendations are subsequently performed by selecting a subgroup of users with similar characteristics to the active user and using their ratings to produce a prediction for the active user. Neighbourhood-based method is also widely called memory-based approach. Model-based technique on the other hand provides recommendations by building models using machine learning and data mining techniques. The model-based technique builds intelligent models by learning and recognizing collection of users' ratings pattern. The intelligent models are eventually used to generate rating predictions of new set of data. 
CF recommender systems in general requires users' or items' past ratings to be able to perform its recommendation function excellently, however there are situations when a user past ratings are not available because the user may not have rated any item as in the case of a new user that requires recommendation. These situations are generally referred to as cold start problems (Revathy \& Anitha, 2019). Cold start problems can be classified into two basic categories depending on the situation: the user cold start problem and the item cold start problem (Zhu et al., 2020). The user cold start problem typically occurs when the RS is faced with the challenge of performing recommendation to a new user because the new user may not have any past record or ratings. Similarly, the item cold start problem arises when a CF recommender system is faced with the task of performing recommendation for a new item it has no information about.

Several applications that make use of recommender systems in suggesting items and users of interest to their users generally make provision for users to create an explicit model of themselves by inputting their demographic data such as age, gender, occupation, place of residence etc. and other relevant information into the systems. Demographic data can be used for neighbourhood selection by computing similarity between the explicit models of users based on the general intuition that most often people with same demographic attribute share similar taste or preferences for similar items. However, it is important to point out that similarity does not necessarily imply predictability even though a relationship can be established between the two. The purpose of recommender systems is to anticipate users' rating for an unseen item. Not all similar users will have the same rating or will rate in the same way, it is therefore very important to measure the predictability of similar users rating in determining their applicability in estimating a new user rating for an item. In view of this, this paper attempts to find the solution to the user cold start problem by using demographic data as additional sources together with existing users' rating and employing entropy-based methodology to determine the degree of predictability.

For the rest of the paper, relevant literature was reviewed in the next section. Section 3 discusses the methodology used in this work with emphasis on the designed algorithm and the experimentation procedure. Section 4 discusses the result and section 5 concludes the paper.

\section{LITERATURE REVIEW}

To solve the cold start problem, various methods have been proposed and used. These methods can be classified under three categories: (i) use of additional data sources rather than users' past ratings (Cantador et al., 2015) (ii) choosing the most prominent group of similar users (Park et al., 2006) (iii) use of hybrid methods (Passi, Jain \& Singh, 2019; Son, 2014). Different authors have attempted solving the cold-start problem. Rashid et al (2002) presented the most popular approach which uses item's popularity in solving the cold-start problem. The items are ranked based on the number of ratings attributed to them by all users and the most popular items are recommended to the new user. Since most users are likely to rate popular items high, ratings may be uninformative because popular items continue to gather more point making not so popular items that may likely be liked by user not to ever be recommended to the user.

Chen et al. (2003) approaches the problem using social network theory and ontological decision model that consider historical ratings information and user ontology information (users' static attributes e.g., age, sex). In their approach, a user to user's relationship network is established based on historical ratings of existing users using a social network theory. This relationship network is used to create multiple sub-communities dependent on the strength of the relationship. A classification decision tree is used to train an ontological decision-making model using the sub-communities and ontological properties of existing users, and is eventually used to place a new user into a sub-community based on the new user's ontology to get similar neighbours. Popular items in the subcommunity are eventually recommended to the new user. This approach did not estimate new user rating for an item, it only assumes that popular items in the cluster that the new user belongs to should be liked by him/her. However, a perfect similarity match between two users does not necessarily implies that they will have the same taste. Lai et al. (2011) developed a cloud-based TV programme recommender systems named CPRS. CPRS uses K-means clustering algorithm and K-Nearest neighbour classification technique in recommending TV programmes to users. Users are partitioned into different clusters using K-means clustering algorithm based on available users' demographics. For a new user of the system with no rating history, the K-Nearest neighbour method is used to add such user into a cluster. Depending on this predicted cluster that the user is added to, popular items in the cluster and other similar clusters are recommended to the active user.

Safoury \& Salah (2013) also used a demographic based solution to attempt the cold start challenge. In their approach, existing users' demographic data together with a new user's demographic data were used to compute similarity between the existing users and the new user and these similarities are subsequently used to determine a neighbourhood for the new user. The frequency of all item's ratings by users in each neighbourhood are computed and items which have been commonly positively-rated by the neighbourhood are suggested to the target (new) user. However, as mentioned earlier, similarity does not necessarily translate to predictability. It is possible to have similar users differing in their ratings of items. Catering for noisy users in the neighbourhood is not taken into consideration. Hawashin et al. (2018) attempted solving the cold start problem by first using the demographic attributes of a target user to determine the group the user belongs to. Furthermore, the actual interests of the group are used to determine the items to be suggested to the intended user. Though the approach looks interesting however it cannot be considered as a pure CF method as the terms that describe the items in the RS are used in determining the interests of users in a group. Al-Bakri \& Hassan (2019) used a clustering 
approach to solve the cold start problem in CF by using fuzzy c-means to analyse user's truthfulness information.

In order to address the gaps highlighted, this paper will combine demographic data as additional sources with existing users' rating and employ entropy-based methodology to determine the degree of predictability in order to estimate ratings for new users.

\section{Methodology}

Three basic stages are involved in recommending item(s) to new users: the data input stage, similarity calculation stage and recommendation calculation stage. Data input stage involves capturing a new user's demographic data as well as the ratings and demographics of existing users. These are stored on and retrieved from database when required. Similarity calculation stage employs users' demographic data gotten from the database to generate a cluster or subgroup of users with similar demographic data to the new user hence forming a neighbourhood. The similarity calculation stage however requires the selection of specific demographic attributes to be employed for calculating the similarities. For example, in a system where demographic attributes such as gender, occupation, country and age are captured, users may only be similar given one or more attributes. User A may be similar to User B based on occupation and similar to user $C$ based on age. As a result, the selected attributes impact the similarity calculation, which also impacts the results of the recommendation calculation.

For the calculation of recommendation, items commonly rated by neighbourhood users are retrieved, then predicted rating values are computed and assigned to the items. The new user is then proposed the items with the highest predicted value. In order to overcome the limitation of existing demographic-based approaches like the work of Rashid et al. (2002) where average item rating over selected attributes is used as predicted rating value, our algorithm extends the average method by considering other factors such as probabilities, degree of disorderliness and significant weight of the attributes selected and consequently factoring these into the item rating prediction computation.

\subsection{ALgORITHM CONSTRUCTION}

When a new user $u$ requires recommendation, the demographic attributes of the user are obtained and for each attribute, an item rating frequency vector/tuple is built that contains frequency of items rated by existing users with similar attribute. For example, if a new user has the attributes shown in Table 1, then all the item ratings for all users that are females are retrieved and an item rating frequency vector is created for each item for the female attribute, the same goes for age and occupation attribute. Table 2 shows an example of item rating frequency vector for the female attribute.

\begin{tabular}{ll}
\multicolumn{2}{c}{ Table 1. User attributes } \\
\hline Gender & $\mathrm{F}$ \\
Age & 23 \\
Occupation & Student \\
\hline
\end{tabular}

Initially item is assigned a rating equal to the most frequent rating (rating with highest number of votes e.g., in Table 2 movie id 1 will be allocated rating 4 because 46 females gave a rating of 4 on movie id 1 ). If item rating to be predicted and most frequent rating are denoted by $r$ and $m f r$ respectively then:

$$
r=m f r
$$

In some situations, more than 1 rating may have the most frequent value, when this occurs, the average rating is used i.e., rating is assigned the average of the most frequent ratings. Next, the popularity of the rating is considered.

Table 2. Sample of female gender movies frequencies

\begin{tabular}{llllll}
\hline Movie id & F1 & F2 & F3 & F4 & F5 \\
\hline 1 & 3 & 8 & 24 & 46 & 28 \\
2 & 1 & 1 & 5 & 8 & 2 \\
3 & 3 & 3 & 3 & 3 & 1 \\
4 & 2 & 5 & 12 & 18 & 3 \\
5 & 0 & 3 & 2 & 13 & 3 \\
\hline
\end{tabular}

Table 3. Item rating frequency vector example 1

\begin{tabular}{llllll}
\hline Rating & 1 & 2 & 3 & 4 & 5 \\
Frequency & 0 & 0 & 40 & 0 & 0 \\
\hline
\end{tabular}

Table 4. Item rating frequency vector example2

\begin{tabular}{llllll}
\hline Rating & 1 & 2 & 3 & 4 & 5 \\
Frequency & 0 & 0 & 40 & 0 & 4 \\
\hline
\end{tabular}

In both Table 3 and 4 , it can be seen that the rating with the most frequent rating which is 40 is rating 3 i.e., rating 3 is the popular rating, however the strength of their popularity differs. The consistency exhibited in Table 3 is more ascertained than Table 4 due to the rating 5 in Table 4 that has a vote or frequency of 4 . Thus, the popularity of rating 3 in Table 3 is stronger than in Table 4 . The difference in popularity or the inconsistencies added to an otherwise consistent state can be regarded as noise $\epsilon$ and this should be subtracted from the initial item rating. So, subtracting $\in$ from equation 1 gives:

$$
r=m f r-\epsilon
$$

The popularity can also be related to predictability i.e., the more the popularity of the rating the higher the predictability which implies more certainty about the outcome of an event. Popularity or consistency or more technically the predictability can be determined using entropy (Jaynes, 1957), which is widely used in information theory. If the probabilities of all the events that may occur in a situation are provided, entropy can be used to compute the degree of uncertainty in such situation. For a chance variable $x$ with $n$ possible associated events, whose probabilities of occurrence are $p_{1}, p_{2}, \ldots, p_{n}$, then the entropy of the chance variable (denoted by $H(x)$ ) is the entropy of the set of probabilities $p_{1}, p_{2}, \ldots, p_{\mathrm{n}}$ and is given by the equation:

$$
H(x)=-\sum p(x) \log p(x)
$$


Entropy range is typically denoted by $0 \leq$ Entropy $\leq$ $\log (n)$, where $n$ is number of outcomes. When one of the probabilities is 1 and the others are zeros (0), the Entropy value is 0 (minimum entropy). Entropy value $\log (n)$ (maximum entropy) occurs when all the probabilities have the same values of $1 / n$. For example, computing entropy for the item rating frequency vector in Table 3, where rating frequencies are the set of probabilities for each rating, an entropy value of 0 is returned which shows that the rating frequency vector is very consistent and predictable i.e., if a new rating should be assigned to the item associated with the item rating frequency vector in Table 3, chances are very high that the rating will be 3 .

On the other hand, computing the entropy for the item rating frequency vector shown in Table 5, maximum entropy value is returned which is characterized by a high (maximum) level of inconsistency and unpredictability since the probability for all the ratings are equal $(1 / 40)$. For example, if a rating should be allocated to an item associated with the item rating frequency vector in Table 5 , chances are very high that the rating will be stuck. It will be very difficult to determine the rating that will be selected. In this case the average of the rating frequency vector is computed and assigned to item rating $r$.

\begin{tabular}{llllll} 
Table 5: Item rating frequency vector example 3 \\
\hline Rating & 1 & 2 & 3 & 4 & 5 \\
Frequency & 40 & 40 & 40 & 40 & 40
\end{tabular}

When the entropy value is neither minimum nor maximum, i.e., lies in between 0 and maximum value as it could occur when entropy is computed for the item rating frequency vector shown in Table 4, the rating should be somewhere between the most frequent rating and the average rating. In this situation, the difference between the most frequent rating and average rating is used as a bias like this: if the most frequent rating is greater than the average rating, the inconsistency or entropy value regarded as noise is subtracted from the most frequent rating as the rating should move closer to the average from the most frequent rating and vice versa. Equation (2) thus becomes

$$
r=m f r \pm \epsilon
$$

The frequency or number of votes is considered next. The more the available data, the much better it is to make an accurate decision. Item rating frequency vectors with higher number of votes are considered to be more informative. Each vector is assigned a significance weight based on the total number of votes or frequency by computing the ratio of number of votes for each vector over all available attributes' vectors using equation 5

$$
w_{v a}=v_{f a} /\left(v_{f a 1}+v_{f a 2}+\ldots+v_{f a n}\right)
$$

where $w_{v a}$ is the ratio/significance weight for item rating frequency vector $v_{f a}$ for attribute $a$. The significance weight is eventually multiplied by the computed rating $r$ for each item as shown in equation 6

$$
r=r_{v a}^{*} w_{v a}
$$

where $r_{v a}$ is the computed rating for item rating frequency vector $v$ for attribute $a$ and $w_{v a}$ is the significance weight of vector $v$ for attribute $a$. Finally, to compute the predicted rating for each item, the computed item rating value for each attribute are summed together as shown in equation 7

$$
p_{r i}=r_{g i}+r_{a i}+r_{o i}
$$

where $p_{\mathrm{ri}}$ is the final rating value predicted for item $i$ and $r_{g i}, r_{a i}$ and $r_{o i}$ are the respective computed rating for attributes gender, age and occupation for item $i$ viz-a-viz a user $u$ user's truthfulness information.

Table 6. Demographic and Entropy Based CF (DEBCF) Recommender algorithm

Input: $A$ (new user demographic attributes), $R$ (existing users' item ratings), $I$ (items), $n$ (maximum rating allowed)

Output: $S$ (new user predicted rating for all items)

1. Get $R M$ (item Rating Frequency Matrix)

2. Initialize an array $S$

3. Foreach $i \in \mathrm{I}$ in $R M$

4. Initialize $S_{i}=0$

5. set $T$ (totalRating of an item for all attributes) $=\sum$ iarfo in I //iarfo is the item attribute rating frequency vector

Foreach $a$ in $A$

Get iarfv (item attribute RatingFrequencyVector)

$D_{i a}($ InitialPrediction $)=\operatorname{mostFrequentRating}($ iarfv $)$

ent $=\operatorname{entropy}($ iarfv $)$

If $e n t=\log (n)$

average $=\operatorname{mean}($ iarfo $)$

$$
D_{\text {ia }}=\text { average }
$$

Else if ent $>0$ \&\& ent $<\log (n)$

$D_{i a}=$ entropyBiasedRatingPrediction $($ iarfv, ent,$D)$

compute $W_{i a}$ (significantWeight) = T/iarfo

$P_{i a}($ prediction $)=W_{i a} * D_{i a}$

$S_{i}=S_{i}+P_{i a}$

End Foreach

End Foreach

Return $S$

\subsection{EXPERIMENTATION}

MovieLens data set ${ }^{1}$, provided by GroupLens movie recommender system, was used for experimentation. The particular groupLens dataset used for the experimentation is MovieLens $100 \mathrm{k}$ and it contains 100,000 ratings on 1682 films from 943 users. Each user had scored at least 20 films, with ratings ranging from 1 (bad) to 5 (excellent). Since the focus of this paper is on tackling a new user recommendation problem, leave-Nout cross validation approach is used for evaluating our algorithm. The performance metrics (Herlocker et al 2004) used for evaluation are: prediction accuracy using Root Mean Square Error (RMSE) and Mean Absolute Error (MAE); and classification metrics using precision and recall. MAE compares the predicted rating to the true rating and compute the average of all the absolute value differences of both ratings. RMSE on the other hand does

${ }^{1}$ http://www.grouplens.org/node/73. 
not use the absolute value of the differences but rather finds the mean value of the squared differences of both ratings and then compute the square root of the result.

Precision determines how efficient the RS is in recommending relevant items. It finds the percentage of the relevant items out of all the recommended items. Recall measures the ability of the RS to recommend as many relevant items as possible from the set of relevant items in the RS. It does express the percentage of relevant recommended items out of all possible relevant items in the RS. Using these metrics, the performance of the algorithm was compared to two existing algorithms - the Most Popular/Popularity algorithm (a common or popular technique for fixing the cold start issue in recommender system that is also popularly used for benchmarking) (Rashid, et al., 2002) and a demographic based approach algorithm (Safoury \& Salah, 2013) which will be referred to as "Safoury \& Salah" in the result table.

The evaluation was carried out by dividing the 100,000 movieLens rating data into 5 partitions of 20,000 ratings per partition. Then each of the partitions is further split into training and test sets where random number of users was selected from each partition as new users and have their ratings hidden for testing and validation, the remaining users were used for training. The 3 different algorithms were executed on each of the training dataset in the 5 partitions. The overall average results for all the five partitions are presented in the following section.

\section{Result AND Discussion}

Table 7 presents the average of the results of the experiments using the five partitions of the data set. Although the most popular method has the best recall, however our proposed algorithm DEBCF has the best overall precision. The precision of "Safoury \& Salah" and DEBCF algorithm were very close. This could be due to the fact that both of them used the same demographic attribute to form the similar neighbourhood. The result might have been different if different attributes were employed. Since both demographic based approaches have a better precision accuracy, then they can be used when precision is of more importance than recall.

Table 7. Performance Evaluation

\begin{tabular}{cccc}
\hline & $\begin{array}{c}\text { Most } \\
\text { popular }\end{array}$ & $\begin{array}{c}\text { Safoury \& } \\
\text { Salah }\end{array}$ & DEBCF \\
\hline MAE & 0.8957 & 0.9033 & 0.8972 \\
RMSE & 1.1226 & 1.1299 & 1.1235 \\
Precision (\%) & 12.9 & 14.7 & 16.0 \\
Recall (\%) & 41.3 & 37.8 & 39.2 \\
\hline
\end{tabular}

\section{Conclusion}

In this paper, an attempt has been made to solve the new user (cold start) problem by using the existing users' ratings and demographic attributes together with the demographic data of the new user. Using some information theory technique like entropy as a measure of predictability in estimating the new user rating for item has made it possible to reduce the uncertainty inherent in memory-based CF. The results show an improvement in precision accuracy of DEBCF algorithm as compared to that of the existing algorithms.

\section{REFERENCES}

Adomavicius, G., \& Tuzhilin, A. (2005). Toward the next generation of recommender systems: A survey of the state-of-the-art and possible extensions. IEEE transactions on knowledge and data engineering, 17(6), 734-749.

AL-Bakri N. L., \& Hassan, S. (2019). A Proposed Model to Solve Cold Start Problem using Fuzzy User-Based Clustering. 2nd Scientific Conference of Computer Sciences (SCCS), 121 -125. Baghdad, Iraq: IEEE.

Amatriain, X., Pujol, J. M., \& Oliver, N. (2009). I like it... i like it not: Evaluating user ratings noise in recommender systems. UMAP 2009, pp. 247-258. Berlin: Springer.

Bell, R., Koren, Y., \& Volinsky, C. (2007). Modeling relationships at multiple scales to improve accuracy of large recommender systems. the 13th ACM SIGKDD international conference on Knowledge discovery and data mining, 95-104

Cantador, I., Fernández-Tobías, I., Berkovsky, S., \& Cremonesi, P. (2015). Cross-Domain Recommender Systems. In F. RicciLior, L. Rokach, \& B. Shapira (Eds.), Recommender Systems Handbook. Springer, Boston, MA.

Chen, M., Yang, C., Chen, J., \& Yi, P. (2003). A Method to Solve Cold-Start Problem in Recommendation System based on Social Network Subcommunity and Ontology Decision Model. 3rd International Conference on Multimedia Technology. doi:https://doi.org/10.2991/icmt-13.2013.20

Hawashin, B., Mansour, A., Kanan, T., \& Fotouhi, F. (2018). An efficient cold start solution based on group interests for recommender systems. Proceedings of the First International Conference on Data Science, E-learning and Information Systems (pp. 1-5). Maldrid, Spain: ACM.

Herlocker, J. L., Konstan, J. A., Terveen, L. G., \& Riedl, J. T. (2004). Evaluating collaborative filtering recommender systems. ACM Transactions on Information Systems (TOIS), 22(1), 5-53.

Herlocker, J., Konstan, J. A., \& Riedl, J. (2002). An empirical analysis of design choices in neighborhood-based collaborative filtering algorithms. Information retrieval, 5(4), 287-310.

Jaynes, E. T. (1957). Information theory and statistical mechanics. Physical review, 106(4), 620-630.

Lai, C.-F., Chang, J.-H., Hu, C.-C., Huang, Y.-M., \& Chao, H.-C. (2011). CPRS: A cloud-based program recommendation system for digital TV platforms. Future Generation Computer Systems, 27(6), 823-835.

Melville, P., \& Sindhwani, V. (2017). Recommender Systems. In C. Sammut, \& G. I. Webb (Eds.), Encyclopedia of Machine Learning and Data Mining. Springer, Boston, MA.

Odumuyiwa, V., \& Oyeyemi, O. (2019). Semantic Enabled Profile Recommender for Social Coding Platform. the 3rd international conference on Transition from Observation to Knowledge to Intelligence (TOKI 2019), 1-11. Lagos, Nigeria.

Park, S., Pennock, D., Madani, O., Good, N., \& DeCost, D. (2006). Naïve filterbots for robust cold-start recommendations. 12th ACMSIGKDD International Conference on Knowledge Discovery and Data Mining (pp. 699-705). ACM. doi:https://doi.org/10.1145/1150402.115

Passi, R., Jain, S., \& Singh, P. K. (2019). Hybrid Approach for Recommendation System. Proceedings of the 2 nd International Conference on Data Engineering and Communication Technology. 828. Springer.

Rashid, A. M., Albert, I., Cosley, D., Lam, S. K., McNee, S. M., Konstan, J. A., \& Riedl, J. (2002). Getting to know you: learning new user preferences in recommender systems. the 7 th international conference on Intelligent, 127-134

Revathy, V. R., \& Anitha, P. S. (2019). Cold Start Problem in Social Recommender Systems: State-of-the-Art Review. In S. K. Bhatia, S. Tiwari, K. K. Mishra, \& M. C. Trivedi (Ed.), Advances in Computer Communication and Computational Sciences. 759, pp. 105-115. Springer.

Safoury, L., \& Salah, A. (2013). Exploiting User Demographic Attributes for Solving Cold-Start Problem in Recommender System. Lecture Notes on Software Engineering, 1(3), 303-307.

Son, L. H. (2014). HU-FCF: a hybrid user-based fuzzy collaborative filtering method in recommender systems. Expert Systems with Applications: An International, 41(15), 6861-6870.

Zhu, Y., Lin, J., He, S., Wang, B., Guan, Z., Liu, H., \& Cai, D. (2020). Addressing the item cold-start problem by attribute-driven active learning. IEEE Transactions on Knowledge and Data Engineering, 32(4), 631-644. 